\title{
Arithmetic of Catalan's constant and its relatives
}

\author{
Wadim Zudilin ${ }^{1}$
}

Received: 24 April 2018 / Published online: 27 May 2019

(c) The Author(s) 2019

\begin{abstract}
We prove that at least one of the six numbers $\beta(2 i)$ for $i=1, \ldots, 6$ is irrational. Here $\beta(s)=\sum_{k=0}^{\infty}(-1)^{k}(2 k+1)^{-s}$ denotes Dirichlet's beta function, so that $\beta(2)$ is Catalan's constant.
\end{abstract}

Keywords Irrationality · Catalan's constant - Dirichlet's beta function · Hypergeometric series

Mathematics Subject Classification $11 \mathrm{~J} 72 \cdot 11 \mathrm{Y} 60 \cdot 33 \mathrm{C} 20$

\section{Introduction}

In this note we discuss arithmetic properties of the values of Dirichlet's beta function

$$
\beta(s)=\sum_{n=1}^{\infty} \frac{\left(\frac{-4}{n}\right)}{n^{s}}=\sum_{k=0}^{\infty} \frac{(-1)^{k}}{(2 k+1)^{s}}
$$

at positive even integers $s$. The very first such beta value $\beta(2)$ is famously known as Catalan's constant; its irrationality remains an open problem, though we expect the number to be irrational and transcendental. The best known results in this direction were given by T. Rivoal and this author in [4]. Namely, we showed that at least one of the seven numbers $\beta(2), \beta(4), \ldots, \beta(14)$ is irrational, and that there are infinitely many irrational numbers among the even beta values $\beta(2), \beta(4), \beta(6), \ldots$ Here we use a variant of the method from $[3,8]$ to improve slightly on the former achievement; a significant strengthening towards the infinitude result, based on a further development of the ideas in [2,6], is a subject of the recent preprint [1] of S. Fischler.

To Peter Bundschuh, with many irrational wishes, on the occasion of his 80th birthday.

\section{Communicated by Jens Funke.}

$凶$ Wadim Zudilin

w.zudilin@math.ru.nl

http://www.math.ru.nl/ wzudilin

1 Department of Mathematics, IMAPP, Radboud University, PO Box 9010, 6500 GL Nijmegen,

Netherlands 
Theorem 1 At least one of the six numbers

$$
\beta(2), \beta(4), \beta(6), \beta(8), \beta(10), \beta(12)
$$

is irrational.

In Sect. 2 we illustrate principal ingredients of the method in a particularly simple situation; this leads to a weaker version of Theorem 1, namely, to the irrationality of at least one number $\beta(2 i)$ for $i=1, \ldots, 8$. The details about the general construction of approximating forms to even beta values and our proof of Theorem 1 are given in Sect. 3 .

\section{Outline of the construction}

For an odd integer $s \geq 3$ (which we eventually set to be 17) and even $n>0$, define the rational function

$$
R_{n}(t)=\frac{2^{6 n} n !^{s-3}(2 t+n) \prod_{j=1}^{3 n}\left(t-n+j-\frac{1}{2}\right)}{\prod_{j=0}^{n}(t+j)^{s}}
$$

and assign to it the related sequence of quantities

$$
r_{n}=\sum_{\nu=1}^{\infty} R_{n}\left(v-\frac{1}{2}\right)(-1)^{v}=\sum_{\nu=n+1}^{\infty} R_{n}\left(v-\frac{1}{2}\right)(-1)^{\nu} .
$$

The sums $r_{n}$ are instances of generalized hypergeometric functions, for which we can use some standard integral representations to write

$$
r_{n}=\frac{2^{6 n}(3 n+1) !}{n !^{3}} \int \cdots \int_{[0,1]^{s}} \frac{\left(1-t_{1} t_{2} \cdots t_{s}\right) \prod_{j=1}^{s} t_{j}^{n-1 / 2}\left(1-t_{j}\right)^{n} \mathrm{~d} t_{j}}{\left(1+t_{1} t_{2} \cdots t_{s}\right)^{3 n+2}}
$$

(details are given in Lemma 2 below). This form clearly implies that $r_{n}>0$ and also gives access to the asymptotics

$$
\lim _{n \rightarrow \infty} r_{n}^{1 / n}=2^{6} 3^{3} \max _{t \in[0,1]^{s}} \frac{\prod_{j=1}^{s} t_{j}\left(1-t_{j}\right)}{\left(1+t_{1} t_{2} \cdots t_{s}\right)^{3}}=12^{3} \max _{0<t<1} \frac{t^{s}(1-t)^{s}}{\left(1+t^{s}\right)^{3}} .
$$

An important ingredient of the construction is the following decomposition of the quantities $r_{n}$.

Lemma 1 For odd $s$ and even $n$ as above,

$$
r_{n}=\sum_{\substack{i=1 \\ i \text { even }}}^{s} a_{i} \beta(i)+a_{0},
$$

where $a_{i}=a_{i, n}$ satisfy the inclusions $\Phi_{n}^{-1} d_{n}^{s-i} a_{i} \in \mathbb{Z}$ for $i=0,1, \ldots$, s even. Here $d_{n}$ denotes the least common multiple of the numbers $1,2, \ldots, n$, and

$$
\Phi_{n}=\prod_{2 \sqrt{n}<p \leq n} p^{\varphi_{0}(n / p)} \quad \text { with } \varphi_{0}(x)= \begin{cases}0 & \text { if } 0 \leq\{x\}<\frac{1}{3}, \\ 1 & \text { if } \frac{1}{3} \leq\{x\}<\frac{1}{2}, \\ 2 & \text { if } \frac{1}{2} \leq\{x\}<1,\end{cases}
$$

the product taken over primes. 
Note that from the prime number theorem we deduce the asymptotics

$$
\lim _{n \rightarrow \infty} d_{n}^{1 / n}=e \quad \text { and } \quad \lim _{n \rightarrow \infty} \Phi_{n}^{1 / n}=e^{\varkappa},
$$

where

$$
\varkappa=\left(\psi\left(\frac{1}{2}\right)-\psi\left(\frac{1}{3}\right)-1\right)+2 \cdot\left(\psi(1)-\psi\left(\frac{1}{2}\right)-1\right)=0.9411124762 \ldots,
$$

the function $\psi(x)$ denotes the logarithmic derivative of the gamma function.

Remark 1 The analogous construction in [4] makes use of a slightly different rational function than (1), namely, of

$$
\widetilde{R}_{n}(t)=\frac{2^{4 n} n !^{s-2}(2 t+n) \prod_{j=1}^{n}\left(t-n+j-\frac{1}{2}\right) \prod_{j=1}^{n}\left(t+n+j-\frac{1}{2}\right)}{\prod_{j=0}^{n}(t+j)^{s}},
$$

so that

$$
R_{n}(t)=\widetilde{R}_{n}(t) \cdot \frac{2^{2 n} \prod_{j=1}^{n}\left(t+j-\frac{1}{2}\right)}{n !} .
$$

The analogous decomposition of a related quantity $\widetilde{r}_{n}$ assumes the form

$$
\widetilde{r}_{n}=\sum_{\substack{i=1 \\ i \text { even }}}^{s} \widetilde{a}_{i} \beta(i)+\widetilde{a}_{0}
$$

in which the rational coefficients $\widetilde{a}_{i}=\widetilde{a}_{i, n}$ satisfy $\Phi_{n}^{-1} d_{n}^{s-i} \widetilde{a}_{i} \in \mathbb{Z}$ for $i=1, \ldots, s$ even, but $\Phi_{n}^{-1} d_{2 n}^{s} \widetilde{a}_{0} \in \mathbb{Z}$. The appearance of $d_{2 n}^{s}$ as the common denominator in place of $d_{n}^{s}$ changes the scene drastically and leads to weaker arithmetic applications.

Proof of Lemma 1 Following the strategy in [5,8] we can write the function (1) as sum of partial fractions,

$$
R_{n}(t)=\sum_{i=1}^{s} \sum_{k=0}^{n} \frac{a_{i, k}}{(t+k)^{i}}
$$

in which $\Phi_{n}^{-1} d_{n}^{s-i} a_{i, k} \in \mathbb{Z}$ for all $i$ and $k$. Indeed, the rational function (1) is a product of simpler ones

$$
\begin{aligned}
\frac{n !}{\prod_{j=0}^{n}(t+j)} & =\sum_{k=0}^{n} \frac{(-1)^{k}\left(\begin{array}{c}
n \\
k
\end{array}\right)}{t+k}, \\
\frac{2^{2 n} \prod_{j=1}^{n}\left(t-n+j-\frac{1}{2}\right)}{\prod_{j=0}^{n}(t+j)} & =\sum_{k=0}^{n} \frac{(-1)^{n+k}\left(\begin{array}{c}
2 n+2 k \\
2 n
\end{array}\right)\left(\begin{array}{c}
2 n \\
n+k
\end{array}\right)}{t+k}, \\
\frac{2^{2 n} \prod_{j=1}^{n}\left(t+j-\frac{1}{2}\right)}{\prod_{j=0}^{n}(t+j)} & =\sum_{k=0}^{n} \frac{\left(\begin{array}{c}
2 k \\
k
\end{array}\right)\left(\begin{array}{c}
2 n-2 k \\
n-k
\end{array}\right)}{t+k}, \\
\frac{2^{2 n} \prod_{j=1}^{n}\left(t+n+j-\frac{1}{2}\right)}{\prod_{j=0}^{n}(t+j)} & =\sum_{k=0}^{n} \frac{(-1)^{k}\left(\begin{array}{c}
4 n-2 k \\
2 n
\end{array}\right)\left(\begin{array}{c}
2 n \\
k
\end{array}\right)}{t+k}
\end{aligned}
$$


and $2 t+n$; the inclusions $d_{n}^{s-i} a_{i, k} \in \mathbb{Z}$ then follow from [8, Lemma 1]. The cancellation by the factor $\Phi_{n}$ originates from the $p$-adic analysis of the binomial factors entering

$$
a_{s, k}=\frac{(2 n+2 k) !(4 n-2 k) !}{(n+k) !(2 n-k) ! k !^{3}(n-k) !^{3}} \cdot(n-2 k)\left(\begin{array}{l}
n \\
k
\end{array}\right)^{s-3} \text { for } k=0,1, \ldots, n,
$$

and the estimate $\operatorname{ord}_{p} a_{i, k} \geq-(s-i)+\operatorname{ord}_{p} a_{s, k} \geq-(s-i)+\varphi(n / p, k / p)$ for primes in the range $2 \sqrt{n}<p \leq n$, where

$$
\varphi(x, y)=\lfloor 2 x+2 y\rfloor+\lfloor 4 x-2 y\rfloor-\lfloor x+y\rfloor-\lfloor 2 x-y\rfloor-3\lfloor y\rfloor-3\lfloor x-y\rfloor
$$

is a periodic function of period 1 in both $x$ and $y$, and from the inequality

$$
\varphi(x, y) \geq \min _{y \in \mathbb{R}} \varphi(x, y)=\min _{0 \leq y<1} \varphi(x, y)=\varphi_{0}(x)
$$

the details can be borrowed from [4, Sect. 7]. Furthermore, the property $R_{n}(-t-n)=R_{n}(t)$ derived from (1) implies $a_{i, k}=(-1)^{i} a_{i, n-k}$ for $i=1, \ldots, s$ and $k=0,1, \ldots, n$.

Recall that $n$ is even, so that $n / 2=m$ is a positive integer. The summation over $v$ in (2) can also start from $-m-1$ (rather than 1 or $n+1$ ), because the function $R_{n}(t)$ vanishes at all half-integers between $-2 n$ and $n$. Therefore,

$$
\begin{aligned}
r_{n}= & \sum_{\nu=-m-1}^{\infty} R_{n}\left(v-\frac{1}{2}\right)(-1)^{v}=\sum_{i=1}^{s} \sum_{k=0}^{n} a_{i, k} \sum_{\nu=-m-1}^{\infty} \frac{(-1)^{v}}{\left(v+k-\frac{1}{2}\right)^{i}} \\
= & \sum_{i=1}^{s} \sum_{k=0}^{n}(-1)^{k-1} a_{i, k} \sum_{\ell=k-m}^{\infty} \frac{(-1)^{\ell}}{\left(\ell+\frac{1}{2}\right)^{i}} \\
= & \sum_{i=1}^{s} 2^{i} \beta(i) \sum_{k=0}^{n}(-1)^{k-1} a_{i, k}+\sum_{i=1}^{s} \sum_{k=0}^{m-1}(-1)^{k-1} a_{i, k} \sum_{\ell=k-m}^{-1} \frac{(-1)^{\ell}}{\left(\ell+\frac{1}{2}\right)^{i}} \\
& -\sum_{i=1}^{s} \sum_{k=m+1}^{n}(-1)^{k-1} a_{i, k} \sum_{\ell=0}^{k-m-1} \frac{(-1)^{\ell}}{\left(\ell+\frac{1}{2}\right)^{i}},
\end{aligned}
$$

where the rules

$$
\sum_{\ell=k-m}^{\infty}=\sum_{\ell=0}^{\infty}-\sum_{\ell=0}^{k-m-1} \text { if } k>m \quad \text { and } \quad \sum_{\ell=k-m}^{\infty}=\sum_{\ell=0}^{\infty}+\sum_{\ell=k-m}^{-1} \text { if } k<m
$$

were applied. Thus, the rational numbers

$$
a_{i}=2^{i} \sum_{k=0}^{n}(-1)^{k-1} a_{i, k} \text { for } i=1, \ldots, s
$$

satisfy $\Phi_{n}^{-1} d_{n}^{s-i} a_{i} \in \mathbb{Z}$, while for the quantity

$$
a_{0}=\sum_{i=1}^{s} \sum_{k=0}^{m-1}(-1)^{k-1} a_{i, k} \sum_{\ell=k-m}^{-1} \frac{(-1)^{\ell}}{\left(\ell+\frac{1}{2}\right)^{i}}-\sum_{i=1}^{s} \sum_{k=m+1}^{n}(-1)^{k-1} a_{i, k} \sum_{\ell=0}^{k-m-1} \frac{(-1)^{\ell}}{\left(\ell+\frac{1}{2}\right)^{i}}
$$


the inclusion $\Phi_{n}^{-1} d_{n}^{s} a_{0} \in \mathbb{Z}$ follows from noticing that

$$
\begin{aligned}
& d_{n-1}^{i} \sum_{\ell=k-m}^{-1} \frac{(-1)^{\ell}}{\left(\ell+\frac{1}{2}\right)^{i}} \in \mathbb{Z} \text { if } 0 \leq k<m, \\
& d_{n-1}^{i} \sum_{\ell=0}^{k-m-1} \frac{(-1)^{\ell}}{\left(\ell+\frac{1}{2}\right)^{i}} \in \mathbb{Z} \text { if } m<k \leq n .
\end{aligned}
$$

Finally,

$$
\begin{aligned}
a_{i}=2^{i} \sum_{k=0}^{n}(-1)^{k-1} a_{i, k} & =(-1)^{i} 2^{i} \sum_{k=0}^{n}(-1)^{k-1} a_{i, n-k} \\
& =(-1)^{i} 2^{i} \sum_{k=0}^{n}(-1)^{n-k-1} a_{i, n-k}=(-1)^{i} a_{i},
\end{aligned}
$$

so that $a_{i}$ vanish for odd $i$.

Set now $s=17$, in which case we compute from (3) that

$$
\lim _{n \rightarrow \infty} r_{n}^{1 / n}=e^{-16.1123070755 \ldots}
$$

hence the linear forms

$$
\Phi_{n}^{-1} d_{n}^{17} r_{n} \in \mathbb{Z} \beta(2)+\mathbb{Z} \beta(4)+\cdots+\mathbb{Z} \beta(16)+\mathbb{Z}
$$

are positive and tend to 0 as $n \rightarrow \infty$. This implies that the eight numbers $\beta(2), \beta(4), \ldots$, $\beta(16)$ cannot be all rational.

\section{General settings}

A natural way to generalize the construction in Sect. 2 follows the recipe of [4] and [7].

For an odd integer $s \geq 5$, consider a collection $\eta=\left(\eta_{0}, \eta_{1}, \ldots, \eta_{s}\right)$ of integral parameters satisfying the conditions

$$
0<\eta_{j}<\frac{1}{2} \eta_{0} \text { for } j=1, \ldots, s \quad \text { and } \quad \eta_{1}+\eta_{2}+\cdots+\eta_{s} \leq \frac{s-1}{2} \eta_{0},
$$

to which we assign, for each positive integer $n$, the collection

$$
h_{0}=\eta_{0} n+1, \quad h_{j}=\eta_{j} n+\frac{1}{2} \text { for } j=1, \ldots, s .
$$

In what follows, we assume that $h_{0}-1=\eta_{0} n$ is even-the condition that is automatically achieved when $\eta_{0} \in 2 \mathbb{Z}$, otherwise by restricting to even $n$.

Define the rational function

$$
R_{n}(t)=R_{n, \eta}(t)=\gamma_{n} \cdot\left(2 t+h_{0}\right) \frac{(t+1) h_{0}-1}{\prod_{j=1}^{s}\left(t+h_{j}\right)_{1+h_{0}-2 h_{j}}}=R_{n}\left(-t-h_{0}\right),
$$

where

$$
\gamma_{n}=4^{h_{0}-1} \frac{\prod_{j=2}^{s}\left(h_{0}-2 h_{j}\right) !}{\left(h_{1}-\frac{1}{2}\right) !^{2}}
$$


and the (very-well-poised) hypergeometric sum

$$
\begin{aligned}
r_{n} & =r_{n, \eta}=\sum_{\nu=0}^{\infty} R_{n}(v)(-1)^{\nu} \\
& =\gamma_{n} \cdot \frac{\Gamma\left(1+h_{0}\right) \prod_{j=1}^{q} \Gamma\left(h_{j}\right)}{\prod_{j=1}^{q} \Gamma\left(1+h_{0}-h_{j}\right)} s_{+2} F_{s+1}\left(\begin{array}{c}
h_{0}, 1+\frac{1}{2} h_{0}, h_{1}, \ldots, h_{s} \\
\frac{1}{2} h_{0}, 1+h_{0}-h_{1}, \ldots, 1+h_{0}-h_{s}
\end{array} \mid-1\right) .
\end{aligned}
$$

Then [4, Lemma 1] implies the following Euler-type integral representation of $r_{n}$ (see also [4, Lemma 3]).

\section{Lemma 2 The formula}

$$
\begin{aligned}
r_{n}= & \frac{4^{h_{0}-1} \Gamma\left(1+h_{0}\right)}{\Gamma\left(h_{1}+\frac{1}{2}\right)^{2} \Gamma\left(1+h_{0}-2 h_{1}\right)} \\
& \times \int \cdots \int_{[0,1]^{s}} \frac{\prod_{j=1}^{s} t_{j}^{h_{j}-1}\left(1-t_{j}\right)^{h_{0}-2 h_{j}}}{\left(1+t_{1} t_{2} \cdots t_{s}\right)^{h_{0}}} \frac{1-t_{1} t_{2} \cdots t_{s}}{1+t_{1} t_{2} \cdots t_{s}} \mathrm{~d} t_{1} \mathrm{~d} t_{2} \cdots \mathrm{d} t_{s}
\end{aligned}
$$

is valid. In particular, $r_{n}>0$ and

$$
\lim _{n \rightarrow \infty} r_{n}^{1 / n}=\frac{\left(4 \eta_{0}\right)^{\eta_{0}}}{\eta_{1}^{2 \eta_{1}}\left(\eta_{0}-2 \eta_{1}\right)^{\eta_{0}-2 \eta_{1}}} \cdot \max _{t \in[0,1]^{s}} \frac{\prod_{j=1}^{s} t_{j}^{\eta_{j}}\left(1-t_{j}\right)^{\eta_{0}-2 \eta_{j}}}{\left(1+t_{1} t_{2} \cdots t_{s}\right)^{\eta_{0}}} .
$$

Computation of the latter maximum is performed in [4, Sect. 4, Remark], and the result is as follows.

Lemma 3 Assume that $x_{0}$ is a unique zero of the polynomial

$$
x \prod_{j=1}^{s}\left(\left(\eta_{0}-\eta_{j}\right)-\eta_{j} x\right)-\prod_{j=1}^{s}\left(\eta_{j}-\left(\eta_{0}-\eta_{j}\right) x\right)
$$

in the interval $0<x<1$, and set

$$
x_{j}=\frac{\eta_{j}-\left(\eta_{0}-\eta_{j}\right) x_{0}}{\left(\eta_{0}-\eta_{j}\right)-\eta_{j} x_{0}} \text { for } j=1,2, \ldots, s .
$$

Then

$$
\max _{t \in[0,1]^{s}} \frac{\prod_{j=1}^{s} t_{j}^{\eta_{j}}\left(1-t_{j}\right)^{\eta_{0}-2 \eta_{j}}}{\left(1+t_{1} t_{2} \cdots t_{s}\right)^{\eta_{0}}}=\frac{\prod_{j=1}^{s} x_{j}^{\eta_{j}}\left(1-x_{j}\right)^{\eta_{0}-2 \eta_{j}}}{\left(1+x_{1} x_{2} \cdots x_{s}\right)^{\eta_{0}}} .
$$

Arithmetic ingredients of the construction are in line with the strategy used in the proof of Lemma 1. For simplicity we split the corresponding statement into two parts. Define

$$
N=\min _{1 \leq j \leq s}\left\{h_{j}-\frac{1}{2}\right\} \quad \text { and } \quad M=\max \left\{h_{0}-2 N-1, h_{1}-\frac{1}{2}\right\},
$$

and notice that the poles of the rational function (4) are located at the points $t=-k-\frac{1}{2}$ for integers $k$ in the range $N \leq k \leq h_{0}-N-1$.

Lemma 4 The coefficients in the partial-fraction decomposition

$$
R_{n}(t)=\sum_{i=1}^{s} \sum_{k=N}^{h_{0}-N-1} \frac{a_{i, k}}{\left(t+k+\frac{1}{2}\right)^{i}}
$$


of (4) satisfy

$$
a_{i, k}=(-1)^{i} a_{i, h_{0}-1-k}
$$

and

$$
\Phi_{n}^{-1} d_{M}^{s-i} a_{i, k} \in \mathbb{Z}
$$

for $i=1, \ldots, s$ and $N \leq k \leq h_{0}-N-1$, where the product over primes

$$
\Phi_{n}=\prod_{\sqrt{2 h_{0}}<p \leq M} p^{\varphi_{0}(n / p)}
$$

is defined through the 1-periodic functions

$$
\varphi_{0}(x)=\min _{0 \leq y<1} \varphi(x, y)
$$

and

$$
\begin{aligned}
\varphi(x, y) & =\left\lfloor 2\left(\eta_{0} x-y\right)\right\rfloor+\lfloor 2 y\rfloor-\left\lfloor\eta_{0} x-y\right\rfloor-\lfloor y\rfloor-2\left\lfloor\eta_{1} x\right\rfloor-\left\lfloor\left(\eta_{0}-2 \eta_{1}\right) x\right\rfloor \\
& +\sum_{j=1}^{s}\left(\left\lfloor\left(\eta_{0}-2 \eta_{j}\right) x\right\rfloor-\left\lfloor y-\eta_{j} x\right\rfloor-\left\lfloor\left(\eta_{0}-\eta_{j}\right) x-y\right\rfloor\right) .
\end{aligned}
$$

Proof For this, we write the function $R_{n}\left(t-\frac{1}{2}\right)$ as the product of $2 t+h_{0}-1$, the three integer-valued polynomials

$$
\frac{4^{h_{1}^{*}}\left(t+\frac{1}{2}\right)_{h_{1}^{*}}}{h_{1}^{*} !}, \frac{4^{h_{0}-2 h_{1}}\left(t+h_{1}^{*}+\frac{1}{2}\right)_{h_{0}-2 h_{1}}}{\left(h_{0}-2 h_{1}\right) !}, \frac{4^{h_{1}^{*}}\left(t+h_{0}-h_{1}^{*}-\frac{1}{2}\right)_{h_{1}^{*}}}{h_{1}^{*} !},
$$

where $h_{1}^{*}=h_{1}-\frac{1}{2}=\eta_{1} n$, and the rational functions

$$
\frac{\left(h_{0}-2 h_{j}\right) !}{\left(t+h_{j}-\frac{1}{2}\right)_{1+h_{0}-2 h_{j}}} \text { for } j=1, \ldots, s \text {. }
$$

Then $[4$, Lemmas $4,5,10,11]$ and the Leibniz rule for differentiating a product imply the inclusions $d_{M}^{s-i} a_{i, k} \in \mathbb{Z}$ and estimates

$$
\operatorname{ord}_{p} a_{i, k} \geq-(s-i)+\varphi\left(\frac{n}{p}, \frac{k}{p}\right)
$$

for the $p$-adic order of the coefficients. These are combined to conclude with (7).

The property (6) follows from the symmetry of the rational function (4).

Lemma 5 The decomposition

$$
r_{n}=\sum_{\substack{i=1 \\ i \text { even }}}^{s} a_{i} \beta(i)+a_{0} \in \mathbb{Q} \beta(2)+\mathbb{Q} \beta(4)+\cdots+\mathbb{Q} \beta(s-1)+\mathbb{Q}
$$

takes place, where $\Phi_{n}^{-1} d_{M}^{s-i} a_{i} \in \mathbb{Z}$ for $i=0,1, \ldots$, s even, and $\Phi_{n}$ is defined in Lemma 4 .

Proof Since the function (4) vanishes at $t=-1,-2, \ldots,-h_{0}+2$, we can shift the summation in (5):

$$
r_{n}=\sum_{\nu=-\left(h_{0}-1\right) / 2}^{\infty} R_{n}(\nu)(-1)^{\nu}=\sum_{i=1}^{s} \sum_{k=N}^{h_{0}-N-1}(-1)^{k} a_{i, k} \sum_{\nu=-\left(h_{0}-1\right) / 2}^{\infty} \frac{(-1)^{\nu+k}}{\left(v+k+\frac{1}{2}\right)^{i}} .
$$


Now proceeding as in the proof of Lemma 1 we arrive at the desired decomposition (8) with

$$
\begin{aligned}
a_{0}= & \sum_{i=1}^{s} \sum_{k=N}^{\left(h_{0}-3\right) / 2}(-1)^{k} a_{i, k} \sum_{\ell=k-\left(h_{0}-1\right) / 2}^{-1} \frac{(-1)^{\ell}}{\left(\ell+\frac{1}{2}\right)^{i}} \\
& -\sum_{i=1}^{s} \sum_{k=\left(h_{0}+1\right) / 2}^{h_{0}-N-1}(-1)^{k} a_{i, k} \sum_{\ell=0}^{k-\left(h_{0}+1\right) / 2} \frac{(-1)^{\ell}}{\left(\ell+\frac{1}{2}\right)^{i}}
\end{aligned}
$$

and

$$
a_{i}=2^{i} \sum_{k=N}^{h_{0}-N-1}(-1)^{k} a_{i, k},
$$

with $a_{i}$ vanishing for $i$ odd in view of the property (6). The inclusions for the coefficients in (8) therefore follow from Lemma 4 and

$$
\begin{aligned}
& d_{h_{0}-2 N-2}^{i} \sum_{\ell=k-\left(h_{0}-1\right) / 2}^{-1} \frac{(-1)^{\ell}}{\left(\ell+\frac{1}{2}\right)^{i}} \in \mathbb{Z} \text { if } N \leq k \leq \frac{h_{0}-3}{2}, \\
& d_{h_{0}-2 N-2}^{i} \sum_{\ell=0}^{k-\left(h_{0}+1\right) / 2} \frac{(-1)^{\ell}}{\left(\ell+\frac{1}{2}\right)^{i}} \in \mathbb{Z} \text { if } \frac{h_{0}+1}{2} \leq k \leq h_{0}-N-1 .
\end{aligned}
$$

Proof of Theorem 1 Take $s=13$ and

$$
\left(\eta_{0}, \eta_{1}, \ldots, \eta_{13}\right)=(31,10,10,10,10,10,11,11,11,11,12,12,12,12),
$$

hence $M=11 n$. Then

$$
\lim _{n \rightarrow \infty} r_{n}^{1 / n}=\exp (-100.73966317 \ldots)
$$

and

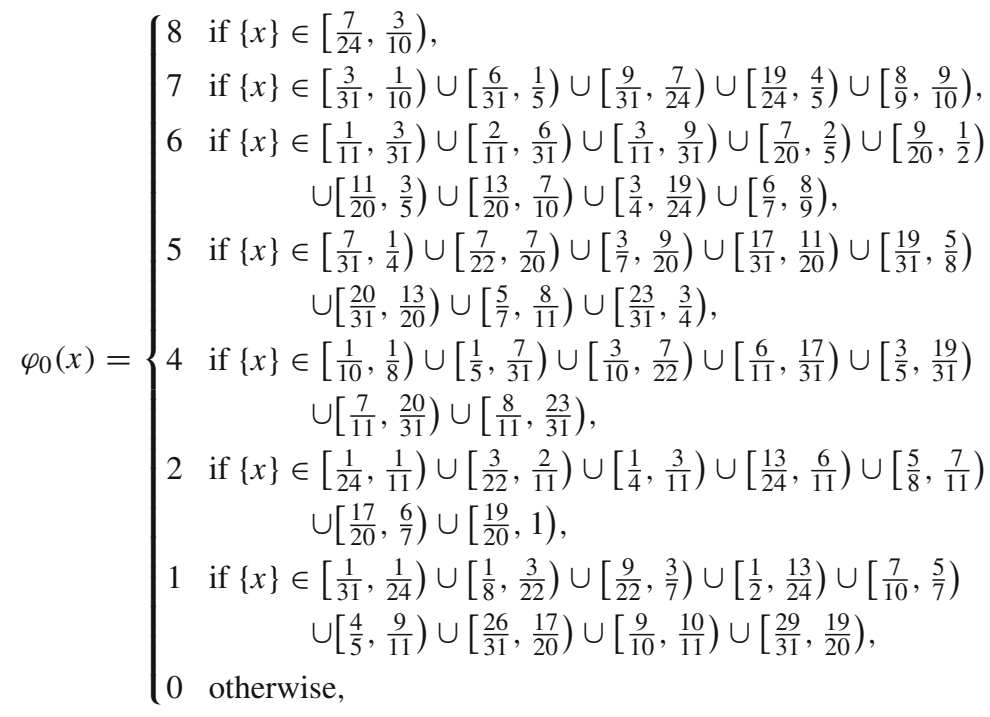


so that

$$
\lim _{n \rightarrow \infty}\left(\Phi_{n}^{-1} d_{M}^{13}\right)^{1 / n}=\exp (100.23354349 \ldots) .
$$

This means that the positive linear forms

$$
\Phi_{n}^{-1} d_{M}^{13} r_{n} \in \mathbb{Z} \beta(2)+\mathbb{Z} \beta(4)+\cdots+\mathbb{Z} \beta(12)+\mathbb{Z}
$$

tend to 0 as $n \rightarrow \infty$. Thus, at least one of the even beta values in consideration must be irrational.

Open Access This article is distributed under the terms of the Creative Commons Attribution 4.0 International License (http://creativecommons.org/licenses/by/4.0/), which permits unrestricted use, distribution, and reproduction in any medium, provided you give appropriate credit to the original author(s) and the source, provide a link to the Creative Commons license, and indicate if changes were made.

\section{References}

1. Fischler, S.: Irrationality of values of $L$-functions of Dirichlet characters. Preprint arXiv: 1904.02402 [math.NT] (2019)

2. Fischler, S., Sprang, J., Zudilin, W.: Many odd zeta values are irrational. Compos. Math. 155(5), 938-952 (2019)

3. Krattenthaler, C., Zudilin, W.: Hypergeometry inspired by irrationality questions. Kyushu J. Math. 73(1), 189-203 (2019)

4. Rivoal, T., Zudilin, W.: Diophantine properties of numbers related to Catalan's constant. Math. Ann. 326(4), 705-721 (2003)

5. Rivoal, T., Zudilin, W.: A note on odd zeta values. Preprint arXiv:1803.03160 [math.NT] (2018)

6. Sprang, J.: Infinitely many odd zeta values are irrational. By elementary means. Preprint arXiv:1802.09410 [math.NT] (2018)

7. Zudilin, W.: Arithmetic of linear forms involving odd zeta values. J. Théor. Nombres Bordeaux 16(1), 251-291 (2004)

8. Zudilin, W.: One of the odd zeta values from $\zeta(5)$ to $\zeta(25)$ is irrational. By elementary means. SIGMA 14, $028(2018)$

Publisher's Note Springer Nature remains neutral with regard to jurisdictional claims in published maps and institutional affiliations. 